Article

\title{
Excluded Volume for Flat Galaxy Rotation Curves in Newtonian Gravity and General Relativity
}

\author{
Rand Dannenberg ${ }^{1,2}$ \\ 1 Optical Physics Company, Simi Valley, CA 93063, USA; rdannenberg@opci.com or rdannenberg@vcccd.edu \\ 2 Physics and Astronomy Department, Ventura College, Ventura, CA 93003, USA
}

Received: 5 February 2020; Accepted: 28 February 2020; Published: 4 March 2020

check for

updates

\begin{abstract}
Using the classical vacuum solutions of Newtonian gravity that do not explicitly involve matter, dark matter, or the gravitational constant, subject to an averaging process, a form of gravity relevant to the flattening of galaxy rotation curves results. The latter resembles the solution found if the vacuum is simply assigned a gravitational field density, and a volume of the vacuum is then excluded, with no averaging process. A rationale then follows for why these terms would become important on the galactic scale. Then, a modification of General Relativity, motivated by the Newtonian solutions, that are equivalent to a charge void, is partially defined and discussed in terms of a least action principle.
\end{abstract}

Keywords: dark matter; dark energy; galaxy rotation curves; alternative gravity theory

\section{Introduction}

$\Lambda C D M$ is the standard cosmological model to which most physicists are committed. However, no dark matter particle has ever been observed, directly or indirectly [1-8]. Important minority alternative relativistic field theories of gravity—such as the Scalar-Vector-Tensor (SVTG) or Modified Gravity (MOG) of Moffat [9], $f(R)$ Gravity [10], the Tensor-Vector-Scalar (TeVeS) gravity of Bekenstein [11], and the non-relativistic Modified Newtonian Dynamics (MOND) of Milgrom [12,13] —are all capable of describing the flattening of galaxy rotation curves without resort to dark matter. MOG introduces multiple additional fields coupled to the Einstein-Hilbert action. TeVeS and MOND modify Newtonian dynamics with acceleration dependent form changes. $f(R)$ gravity modifies General Relativity to include modifiable functions of the Ricci scalar in the Lagrangian. The case for the existence of dark matter is very strong based on astrophysical observations, yet the approaches listed above continue to be investigated because of the lack of direct concrete evidence of dark matter particles (axions, WIMP's).

The addition of a force term going as $1 / r$ in Newtonian gravity to explain the observed flattening is not at all new, for example Kuhn in 1986 and the many references therein as far back as 1963 [14]. It also forms part of the basis for MOND. Rather, it is the explanation of the origin of such a force that is evolving.

The modification of a purely Newtonian force law, with no relativistic features, cannot explain phenomena such as gravitational waves, gravitational lensing and the Bullet Cluster observations (the attachment of lensing effects to the galaxies in the collision and not the gases), redshifts, the CMB and its power spectrum features, BAO, the Tully-Fisher relation, accelerated expansion, inflation, etc. Many alternative theories of gravity have been cast in terms of Parameterized Post-Newtonian (PPN) gravity, but the overall consensus is that none of these theories can simultaneously address all observations without dark matter or energy. Ultimately, a modification to General Relativity would be needed, the same motivations behind the other modified gravity efforts aimed at providing alternative explanations for observations, other than dark matter. None of the modified gravity theories are 
capable of producing all the observations attributed to dark matter, making the case for $\Lambda \mathrm{CDM}$ all the stronger. A different modification scheme to General Relativity will be addressed here, while it should also be realized that it is not realistic to expect to address all observations in a first paper on the suggested scheme.

An explanation for flattened galactic velocity curves will be offered that does not explicitly (or directly) involve any matter or $G$ at all, and depends on voids in spacetime, and assumed gravitational properties of the vacuum.

A Newtonian picture is developed first for insight. In lieu of any perceived lack of physical correctness based on instinct, the true value for the Newtonian model here, is as a seedling idea that leads to a formative description involving a modified form of General Relativity. The central idea is that voids prevent interactions that lead to communicable forces. The particles or fields involved would be in vacuum states, and as such, they are not directly detectable. The idea is in a very weak analogy to the Casimir effect, in which excluded electromagnetic vacuum modes result in a force non-cancellation, with a resultant attractive force between metal plates. As those photons are in vacuum states, one can only indirectly infer their presence by forces measured on the plates.

The solutions to be examined have always been available, starting with the vacuum solutions of classical, non-relativistic gravity. This idea has already been examined by Zhang for a particular solution [15], with a compelling argument for why it is reasonable to add such terms (though without development of the specific source of effects), the most important of which is that the Einstein Field Equations in the weak-field and low-speed limits reduce to the Newtonian Field (potential) Equation (4) below, but not all the way to a particular force law. Therefore, General Relativity does not exclude the unsourced vacuum solutions of Newtonian gravity. Here, solutions different than those considered in [15] will be examined, with spherical and cylindrical symmetry, with the introduction of an averaging process for the spherical case. The source is specifically matter-free, with the homogeneous solutions identified directly with the classical vacuum. A form of charge associated with the vacuum is introduced, along with a mechanism explaining why the charge center is coincident with the presence of a black hole.

The objective here is not to explain away the need for dark energy or dark matter, as the indirect evidence for it supported by observations, and the success of theories utilizing it are so compelling. However, with no direct observations of particles, we owe it to ourselves to examine other explanations, some possibly fringe. The unusual averaging operations needed for the spherical case requires special assumptions to arrive at the needed functional form, and it might be said this investigation further strengthens the need for dark matter, though the investigation is not complete. Following that, a suggestion is made for how to arrive at the needed form without an averaging operation, with an assumption made concerning the gravitational properties of the vacuum by the exclusion of voids that puncture a uniform distribution that would, otherwise, lead to force cancellation. Then a discussion concerning ways to puncture fields is given in terms of General Relativity and a least action principle.

\section{Vacuum Solutions and Averaging Processes}

For a mass density $\rho$, Gauss's Law and the Poisson equation for the Newtonian gravitational field $g$ and potential $\psi$ are,

$$
\begin{gathered}
\nabla \cdot \bar{g}=-4 \pi G \rho \\
\psi=\psi_{\text {mat }}+\psi_{v a c} \\
\bar{g}=-\nabla \psi \rightarrow-\nabla \psi_{m a t}-\nabla \psi_{v a c} \\
\nabla^{2} \psi=\nabla^{2} \psi_{m a t}=4 \pi G \rho
\end{gathered}
$$

Defined this way, the condition that is preserved is,

$$
\nabla \times \bar{g}=0
$$


Therefore, only the divergence of the field is governed by the mass distribution, and the total field can still be described by a potential function. The potential function contains extra terms not governed by the mass that when differentiated once, produce additional field and force terms.

Equation (4) implies the vacuum condition (6), and is satisfied by (7c),

$$
\begin{aligned}
& \nabla^{2} \psi_{v a c}=0 \\
& \psi_{\text {vac }}=\left\{\begin{array}{l}
\sum_{l=0}^{\infty} \sum_{m=0}^{\infty}\left(A_{l} r^{l}+B_{l} r^{-l-1}\right) P_{l}^{m}(\cos \theta)\left(S_{m} \sin (m \varphi)+C_{m} \cos (m \varphi)\right) \\
\sum_{l=0}^{\infty} \sum_{m=0}^{\infty}\left(A_{l} \sinh k z+B_{l} \cosh k z\right) J_{l}(k r)\left(S_{m} \sin (m \varphi)+C_{m} \cos (m \varphi)\right) \\
q \ln (r / a)
\end{array}\right. \\
& \psi_{\text {HOMO }}=\frac{1}{2} \kappa r^{2}-\kappa z^{2} \quad c y l .
\end{aligned}
$$

where the polar angle is $\theta$, the azimuthal angle is $\varphi, S_{m}, C_{m}, A_{l}$ and $B_{l}$ are coefficients, and $P(l, m)$ are the Associated Legendre polynomials. The $J_{l}$ are the Bessel functions, and $k$ is a constant. The quantities $q$ and $a$ are also constants. To explain the flat galaxy rotation curves, normally the vacuum terms are not added, rather, a dark matter density distribution falling off with range as $1 / r^{2}$ or similar function is added to the luminous matter distribution, as this contribution results in a field going as $1 / r$, and a potential going as the logarithm.

Now, consider instead the vacuum solutions, for which there are many. The vacuum term is the homogeneous part of the solution that is never added to the inhomogeneous solutions in the presence of matter, as adding these solutions changes the equations of motion, unlike a gauge symmetry. The solution $\psi_{\text {HOMO }}(8)$ is due to Zhang in cylindrical coordinates with $\kappa$ a constant [15].

The logarithmic cylindrically symmetric coordinate solution is the fundamental solution for the Green's function of the Laplacian on 2D domains. This is exactly the form that is needed to result in a flat rotation curve in the limit of a perfectly flat, disk-like galaxy, and many galaxies have such a structure, especially at the extremity of the luminous mass.

The 3D spherical coordinate solutions as written in (7a) do not admit the form needed to explain a perfectly flat, ideal rotation curve, namely, a logarithmic dependence, but the cylindrical solution (7c) does, automatically. This paper will examine operations on (7a) and (7c) that produce fields going as $1 / r$ in spherical coordinates.

In order to approach the needed logarithmic behavior in a potential with spherical symmetry, an averaging operation will be performed on the vacuum solutions, and different operations are possible. Prior to averaging, the potentials of $(7 \mathrm{a}-\mathrm{c})$ lead to non-zero fields. The vacuum solutions are not pinned to any particular orientation, and all orientations may be treated as being simultaneously active, subject to a weighting.

Unknowns concerning the gravitational aspects of the vacuum will be exploited in the development of an averaging process, as follows:

1. The process is constrained to match the experimental observation that a logarithmic potential is required.

2. Weighting need not be equal for all orientations.

3. Averaging need not utilize the entirety of the surface.

4. Processed solutions for 3D cannot obey the original 3D gravitational vacuum equations.

5. Solutions are built from manipulations of the original vacuum solutions.

Consider the first of three processes. Start with the 2D cylindrical solution, Equation (7c). An averaging process for the potential is examined that allows the 2D solution to be raised to 3D. Imagine that there is a $3 \mathrm{D}$ radial vector from the origin, and one averages over the value of the intersection point of the radial vector with the cylinder, for all possible orientations of the cylinder rotated about the origin. The radii of interest in this case ranges from the smallest to the largest components of the 
radial vector. Equally weighting all of the orientations would not produce the desired result. The weighting must be such that only orientations of the cylinder that are perpendicular to its sidewall be counted, and this equates to the 2D logarithmic solution operating in 3D. The 3D average remains a vacuum solution in $2 \mathrm{D}$, from which it was derived,

$$
\left\langle\psi_{v a c}\right\rangle=\left\langle\psi_{v a c}^{2 D}\right\rangle=\psi_{v a c}^{2 D}=q \ln (r / a)
$$

Now consider the 3D spherical solutions (7a). When the orientations are equally weighted, the operation is equivalent to averaging over a surface. Consider first the average of a divergence-less source for the field over an arbitrary surface $A$, including that of a sphere,

$$
\begin{gathered}
\nabla \cdot \bar{g}_{v a c}=0 \\
\left\langle\bar{g}_{v a c}\right\rangle_{A}=\frac{1}{A} \int_{A} \bar{g}_{v a c} \cdot d \bar{A}=\frac{1}{A} \int_{V} \nabla \cdot \bar{g}_{v a c} d V=\frac{1}{A} \int_{V} \nabla^{2} \psi_{v a c} d V=0
\end{gathered}
$$

Clearly, for this type average of the field is zero, and does not lead to the needed functional form.

Consider a second process where the potential is averaged over $A$ for a spherical surface and azimuthal symmetry with $m=0$. One finds that due to the $\sin \theta$ term in the integrand, when integrating over a spherical surface, only the $l=0$ term is non-zero,

$$
\begin{gathered}
\left\langle\psi_{\text {vac }}^{m=0}\right\rangle_{A}=\frac{1}{A} \int_{A} \psi_{\text {vac }}^{m=0} d A=A_{0}+\frac{B_{0}}{r} \\
\left\langle\psi_{\text {vac }}\right\rangle=\frac{1}{\zeta_{o}} \int_{a}^{r}\left\langle\psi_{\text {vac }}^{m=0}\right\rangle_{A} d r=\frac{B_{0}}{\zeta_{o}} \int_{s_{o}}^{s} \frac{d s}{s}=q \ln \left(s / s_{o}\right)=q \ln (r / a)
\end{gathered}
$$

where the coefficients have absorbed factors from the integration. If one does not demand the second term of (12) be zero, the field is $-B_{o} / r^{2}$, and again, not the needed functional form. The divergence of the field associated with (12) is zero, as so the $3 \mathrm{D}$ average remains a vacuum solution in $3 \mathrm{D}$ from which it was constructed.

Questions are now posed:

1. What is the scale factor of the gravitational vacuum, and is it the same as the scale factor of the universe with matter, radiation, dark energy, and curvature?

2. Is there more than one vacuum, one for each type of particle field, with its own cosmological constant?

3. Can multiple scales in the vacuum be simultaneously active, such that one experiences a sum of potentials at any given position?

The answers to these questions are not at all obvious, although the de Sitter and anti-de Sitter solutions assume there is a single vacuum. The net vacuum potential is therefore taken to be the sum of potentials over some range, suggested in (13), to arrive at the required form. The scale factor of the vacuum $s$ is defined as $r=s x$ with co-moving coordinates $x, A_{0}=0$, and $\zeta_{0}$ is a constant. The upper limit $\mathrm{s}$ is taken to be the scale factor of the universe today.

The third process essentially converts a spherical 3D arrangement into a sampling of a 2D cylindrical cross section through the origin. Assuming azimuthal symmetry again with $m=0$, for an orientation weighting such that only rotation of the $z$-axis through the polar angle in the plane formed by the $z$-axis and radial vector contribute, the entire range of surface values are sampled, yet is 
equivalent to averaging over only polar angle $\theta$, not over area. Thus, the $\sin \theta$ term is eliminated from the integrand, and the even $l$ terms remain. Assuming a spherically symmetric distribution of matter $\rho$,

$$
\begin{gathered}
\left\langle\psi_{\text {mat }}\right\rangle=\psi_{\text {mat }} \\
\left\langle\psi_{\text {vac }}^{m=0}\right\rangle_{\theta}=\sum_{l=0}^{\infty}\left(A_{l} r^{2 l}+B_{l} r^{-2 l-1}\right) \approx q \ln (r / a) \\
\left\langle\bar{g}_{\text {vac }}\right\rangle_{\theta}^{(m=0)}=-\nabla\left\langle\psi_{\text {vac }}^{m=0}\right\rangle_{\theta}=-\frac{q}{r} \hat{r} \\
\nabla \cdot\left\langle\bar{g}_{\text {vac }}\right\rangle_{\theta}^{(m=0)}=-\frac{q}{r^{2}} \\
\nabla \times\left\langle\bar{g}_{\text {vac }}\right\rangle_{\theta}^{(m=0)}=0
\end{gathered}
$$

where the constant values of the Legendre polynomial averages have been absorbed into the coefficients. Neither $G$ nor any enclosed mass enters the solution for the vacuum potential. Terms from the series of Equation (15) may be used to fit a rotation curve, in combination with the known luminous matter densities.

Stated another way, this third process requires the definition of a fixed orbital plane that the $z$-axis is normal to, and the average is over a cross section of a plane through the origin normal to the orbital plane.

For the ideal limit of the perfectly flat rotation curve, one only needs a few of the terms of Equation (15) to make a satisfactory approximation to the logarithm over the finite range of a galaxy. By increasing the number of terms, the agreement may be found to any required precision. Note that the field has acquired a divergence from the averaging process for the two weighted cases, and it is important to point out that that in matter free space, the net field is still zero despite this, developed in Section 3.

In addition, two of the processed cases involve what is essentially a sample of the potential reduced by one dimension. The significance of that may, or may not, relate to the holographic principle, in which all information relevant to existence in $N$ dimensions is contained in $N-1$ dimensions. The dimension reduction leads to a non-zero divergence of the field. The next section will bring black holes and horizons into the picture, to which the holographic principle is closely tied.

Then, for a circular orbit about enclosed mass $M_{\text {enc }}$, a simple approximation for a star at the periphery of a galaxy, with a spherically symmetric matter distribution, and the vacuum term, one finds in the orbital plane,

$$
\begin{gathered}
F_{r}=-m \partial_{r}\left\langle\psi_{m a t}+\psi_{\text {vac }}\right\rangle_{\theta}^{m=0}=-\frac{G m M_{e n c}(r)}{r^{2}}-\frac{m q}{r}=-\frac{m v^{2}(r)}{r} \\
\bar{g}_{q}=-q \frac{\hat{r}}{r} \\
v^{2}(r)=\frac{G M_{e n c}(r)}{r}+q .
\end{gathered}
$$

From (21), the circular velocity becomes a constant $q^{1 / 2}$ for large $r$, and the rotation curve is therefore flat. Note that neither mass nor $G$ explicitly/directly enter $q$.

Any coefficients (or collection of coefficients) in the vacuum solutions that had demonstrated physical importance would be named as a physical quantity. For example, the constant $q$ may be given a name, such as rotary charge, the constant $a$ is the rotary length, and Equation (15) is the rotary potential. The rotary length plays no part in the force term or in the rotary field (20), a re-notated version of (16).

The rotary solutions coincide with the galactic center, and this is rationalized in Section 3, along with why the rotary in field matter free space is actually zero. The rotary parameters would simply be 
inferred from measurement, the same way normal mass is deduced. One might conduct an experiment with clocks or measure a photon redshift with a modified formula from one radius to another within the galaxy. The frequency shift should still be deducible from the gravitational potential difference $\Delta \psi / c^{2}$, and the Equivalence Principle to first order. For sufficiently large radii, the red (or blue) shift is,

$$
\frac{\omega\left(r_{2}\right)}{\omega\left(r_{1}\right)} \approx 1-\frac{G M_{\text {enc }}\left(r_{1}\right)}{r_{1} c^{2}}+\frac{q}{c^{2}} \ln \left(r_{1} / a\right)+\frac{G M_{\text {enc }}\left(r_{2}\right)}{r_{2} c^{2}}-\frac{q}{c^{2}} \ln \left(r_{2} / a\right) \rightarrow 1+\frac{q}{c^{2}} \ln \left(r_{1} / r_{2}\right)
$$

Equation (22) illustrates that the rotary charge is capable of causing frequency shifts, though not explicitly dependent on mass, and also the rotary length $a$ would not have a physical effect to first order. For a typical galaxy $q \sim(100 \mathrm{~km} / \mathrm{s})^{2}$, so $q / c^{2} \sim 10^{-7}$.

The results above constitute an initial rationale for how a gravitational field with a certain form can arise, without resort to dark matter. The processes, though unconventional, are conceptually no more difficult to accept than only indirect evidence of dark matter and energy, the holographic principle, many-worlds theory, multiverse theory. There are undoubtedly other ways to manipulate the vacuum solutions that may be instinctually more palatable. The averaging processes are not critical to the main theme of the paper, as the next section discusses.

\section{Excluded Volume with Assumed Gravitational Property of the Vacuum}

One need not rely on the averaging process of Section 2 at all, if it is simply asserted that the vacuum has a gravitational field density $u_{v a c}$ associated with it, per (24).

As objects are not observed to orbit in matter-free space, it is evident that the net rotary field in matter-free space must be zero. A perfectly uniform, homogeneous, and isotropic rotary charge density $\rho_{q}$ in an infinite domain $\Omega$ then provides a vector cancellation at all points $R$ per (25),

$$
\begin{gathered}
\bar{d}=\bar{R}-\bar{r} \\
\bar{u}_{\text {vac }}(\bar{d})=\rho_{q} \frac{\bar{R}-\bar{r}}{|\bar{R}-\bar{r}|^{2}} \\
\bar{g}_{\Omega}(\bar{R})=\int_{\Omega} \rho_{q} \frac{\bar{R}-\bar{r}}{|\bar{R}-\bar{r}|^{2}} d^{3} \bar{r}=0
\end{gathered}
$$

The domain $\Omega$ will be divided into a spherical domain $S$ centered at the origin, and the remainder is $\Omega-S$,

$$
\bar{g}_{\Omega}(\bar{R})=\underbrace{\int_{S} \rho_{q} \frac{\bar{R}-\bar{r}}{|\bar{R}-\bar{r}|^{2}} d^{3} \bar{r}}_{\bar{g}_{S}}+\underbrace{\int_{\Omega-S} \rho_{q} \frac{\bar{R}-\bar{r}}{|\bar{R}-\bar{r}|^{2}} d^{3} \bar{r}}_{\bar{g}_{\Omega-S}}=0
$$

Evidently,

$$
\begin{gathered}
\bar{g}_{\Omega-S}(\bar{R})=-\bar{g}_{S}(\bar{R}) \\
\bar{g}_{\Omega-S}(\bar{R})=-\int_{S} \rho_{q} \frac{\bar{R}-\bar{r}}{|\bar{R}-\bar{r}|^{2}} d^{3} \bar{r}
\end{gathered}
$$

Equation (28) is the field that would be sensed at the point $R$ inside $\Omega-S$ if the domain $S$ were a missing void. For distances well beyond the boundaries of $S$ when $R \gg r$, Equation (28) simplifies to (29)

$$
\bar{g}_{\Omega-S}=-\bar{g}_{S}=-\rho_{q} V_{S} \frac{\hat{R}}{|\bar{R}|}
$$


which is the same as the attractive rotary field in the notation of (20) shown again,

$$
\bar{g}_{q}=-q \frac{\hat{r}}{r}
$$

The rotary charge and field are therefore identified with a spherical volume at the galactic center that is somehow prevented from interacting. As it is known that there are supermassive blackholes at the centers of galaxies from which particles and light may not escape, the event horizon is a candidate explanation for $V_{S}$, why the rotary charge coincides with the galactic center, and why it would become important only on galactic scales. If the latter is the case, Equations (29) and (30) imply $q=\rho_{q} R_{S}{ }^{3} \propto$ $\rho_{q}\left(G M_{B H} / c^{2}\right)^{3}$, where $R_{S}$ is the Schwarzschild radius, and $M_{B H}$ is the black hole mass. The galaxy circular velocity in the flat region is then from (21) $v \propto M_{B H} 3 / 2$. This volume estimation is simplistic, as it assumes that the density of rotary charge in the vacuum is constant beneath the event horizon, while neglecting aspects concerning the physical volume due to the metric, and unknowns with how the vacuum interacts with it.

The additional force is due to the void caused by the event horizon of the gravitationally collapsed matter, but a distinction is drawn here-it is that the force would be generated by any mechanism that excludes the volume $V_{S}$ from interacting, and in this case, it just happens to be collapsed mass with $G$ and $M_{B H}$ entering indirectly. An excellent overview of void-based astrophysics and cosmology may be found in [16], and other recent work in [17]. Though the references do not deal directly with the impact of collapsed matter on the vacuum, the implication is that voids are relevant for understanding the nature of the vacuum, and dark gravity. The voids in the cases of [16,17] represent regions of space in which dark matter is minimal, and dark energy would dominate.

\section{Modified General Relativity with Excluded Volume}

The last section motivates one to consider formally enforcing the exclusion of a spatial domain in the context of a least action principle, involving a form of modified scalar-tensor gravity with inverse metric $g^{\mu v}$. The exclusion is enforced in the sense that it does not rely solely on the gravitational field arising from a matter distribution. The challenge is to find the right formulation for the exclusion, producing a conserved charge, and not in conflict with any observations. The boundary conditions will be developed in future work, with $S$ taken to be beneath the event horizon of a black hole. Five schemes will be initially sketched below, and they involve either the total contribution to the action from a void being zero, or, the variation of the action in a void being zero, or a field in the void going to zero, or a field becoming discontinuous at the boundary of $S$.

All of the schemes require the generation of a self-consistent solution, in which a horizon coordinate is an input, and the solution must reproduce it as an output. Some require solving a variational problem over a non-infinite or partitioned domain. Some require a symmetry of the Lagrangian. New techniques will have to be developed for solving the equations, and whether the theory can be expressed in the PPN formalism would have to be investigated after. Whether any of these schemes are problematic for rotation, or are related to the Kerr metric, or are related to explanations of lensing by twisting universes must also wait until a metric can be derived [18]. There are a large number of theories of alternative gravity, and to determine if the schemes outlined lead to one that has already been examined also requires solutions to be found. The comparisons looking for matches are a great undertaking in themselves.

A very general scalar-tensor model with action $I$, with no cosmological constant, with a scalar field $\beta \varphi$ and $\beta$ a constant, coupling to matter (radiation, mass, any particle fields, electromagnetic fields) in $\mathcal{L}_{m}$, a coupling constant $\omega$, and also incorporating a function of the Ricci scalar $f(R)$ is,

$$
I=\int\left\{\frac{1}{2 \kappa} F(\beta \varphi) f(R)-\omega B(\beta \varphi) g^{\mu \nu} \nabla_{\mu} \varphi \nabla_{v} \varphi-V(\beta \varphi)+Z(\beta \varphi) \mathcal{L}_{m}\left\{M(\beta \varphi), g^{\mu \nu}\right\}\right\} \sqrt{|g|} d^{4} x
$$

where $F, B, Z, V, M$ are functions of the scalar field. 
As a first scheme, for an arbitrary Lagrangian density where the presence of $\{\varphi\}$ is understood to include any derivatives of the scalar field, then dividing the spatial domains per Section 3,

$$
I=\int_{\Omega-S} \mathcal{L}\left\{g^{\mu v}, \beta \varphi\right\} \sqrt{|g|} d^{4} x+\int_{S} \mathcal{L}\left\{g^{\mu v}, \beta \varphi\right\} \sqrt{|g|} d^{4} x
$$

Since the variations with respect to the two fields individually must be zero, and understanding that only the spatial domain is divided, one finds,

$$
\begin{aligned}
& \delta_{g} \int_{\Omega-S} \mathcal{L}\left\{g^{\mu v}, \beta \varphi\right\} \sqrt{|g|} d^{4} x=-\delta_{g} \int_{S} \mathcal{L}\left\{g^{\mu v}, \beta \varphi\right\} \sqrt{|g|} d^{4} x \\
& \delta_{\varphi} \int_{\Omega-S} \mathcal{L}\left\{g^{\mu v}, \beta \varphi\right\} \sqrt{|g|} d^{4} x=-\delta_{\varphi} \int_{S} \mathcal{L}\left\{g^{\mu v}, \beta \varphi\right\} \sqrt{|g|} d^{4} x
\end{aligned}
$$

Continuing the first scheme, let it be asserted that the subdomain $S$ is excluded from interacting in a manner analogous to Section 3, and that manifests as the variations (33a-b) both innately equal to zero, leading to,

$$
\begin{gathered}
\delta_{g} \int_{S} \mathcal{L}\left\{g^{\mu v}, \beta \varphi\right\} \sqrt{|g|} d^{4} x=0 \\
\delta_{\varphi} \int_{S} \mathcal{L}\left\{g^{\mu v}, \beta \varphi\right\} \sqrt{|g|} d^{4} x=0 \\
\delta_{g} \int_{\Omega-S} \mathcal{L}\left\{g^{\mu v}, \beta \varphi\right\} \sqrt{|g|} d^{4} x=0 \\
\delta_{\varphi} \int_{\Omega-S} \mathcal{L}\left\{g^{\mu v}, \beta \varphi\right\} \sqrt{|g|} d^{4} x=0
\end{gathered}
$$

One encounters the complexity of having to solve a variational problem over a non-infinite spatial domain. While (32), (33a-b) and (34a-d) are not the only possible domain divisions, (34d) can lead to a conserved current, and therefore a conserved charge in the domain $S$ if there is a continuous symmetry involving $\varphi$, where the Lagrangian density changes by a total derivative for an arbitrary set of functions $F^{\mu}$, along the lines of,

$$
\begin{aligned}
& \delta_{\varphi}\left(\mathcal{L}\left\{g^{\mu \nu}, \beta \varphi\right\} \sqrt{|g|}\right)=\{\underbrace{\left[\frac{\partial \mathcal{L}}{\partial \varphi}-\partial_{\mu} \frac{\partial \mathcal{L}}{\partial\left(\partial_{\mu} \varphi\right)}\right]}_{0} \delta \varphi+\partial_{\mu}\left(\frac{\partial \mathcal{L}}{\partial\left(\partial_{\mu} \varphi\right)} \delta \varphi\right)\} \sqrt{|g|}=\partial_{\mu} F^{\mu}(\varphi) \sqrt{|g|} \\
& j^{\mu}=\frac{\partial \mathcal{L}}{\partial\left(\partial_{\mu} \varphi\right)} \delta \varphi-F^{\mu}(\varphi) \\
& \partial_{\mu} j^{\mu}=0 \\
& q=\int_{S} j^{0} \sqrt{|g|} d^{3} x \\
& \dot{q}=\int_{S} \partial_{0} j^{0} \sqrt{|g|} d^{3} x=-\int_{S} \partial_{l} j^{l} \sqrt{|g|} d^{3} x=0
\end{aligned}
$$

The result (39) depends on $j$ having zero spatial divergence, or expressed as a surface integral the spatial vector $j$ integrates to zero on the boundary of $S$, or the spatial vector components $j^{l}$ are zero on the boundary of $S$. The Lagrangian must display the symmetry, and may be non-unique. 
The second scheme for the spatial division of the action involves a static field $f_{S}$ and the Schwarzschild radius $R_{S}$,

$$
f_{S}(\bar{r})= \begin{cases}1 & r>R_{S} \\ \varepsilon & r \leq R_{S}\end{cases}
$$

used as follows,

$$
I=\int_{\Omega} f_{S}(\bar{r}) \mathcal{L}\left\{g^{\mu \nu}, \beta \varphi\right\} \sqrt{|g|} d^{4} x
$$

Equations (40) with $\varepsilon=0$ and (41) result in (34c-d) being reproduced, while the contribution to the action of domain $S$ becomes zero, not innately, but due to $f_{S}$, so Equation $(34 a, b)$ are no longer applicable.

For the third scheme, the scalar field becomes $f_{S} \beta \varphi$, the division being that the scalar field becomes zero (or some other value proportional to $\varepsilon$ ) beneath the event horizon,

$$
I=\int_{\Omega} \mathcal{L}\left\{g^{\mu \nu}, f_{S}(\bar{r}) \beta \varphi\right\} \sqrt{|g|} d^{4} x
$$

The fourth scheme is to assert for the event horizon volume, the contribution of domain $S$ to the action is innately zero, not its variation per $(34 a-b)$, and therefore depends on the form of the Lagrangian density,

$$
\int_{S} \mathcal{L}\left\{g^{\mu v}, \beta \varphi\right\} \sqrt{|g|} d^{4} x=0
$$

A candidate action to begin this effort with is (44),

$$
\begin{gathered}
I=\int_{\Omega} \frac{1}{\beta \varphi}\left\{\frac{R-2 \Lambda}{2 \kappa}+\mathcal{L}_{m}\left\{g^{\mu v}, M(\beta \varphi)\right\}\right\} \sqrt{|g|} d^{4} x \\
I=\int_{\Omega} \frac{1}{\beta \varphi}\left\{\frac{R-2 f_{S}(\bar{r}) \Lambda}{2 \kappa}+\mathcal{L}_{m}\left\{g^{\mu v}, M(\beta \varphi)\right\}\right\} \sqrt{|g|} d^{4} x
\end{gathered}
$$

chosen due to its simplicity, and because the scalar field divides the entirety of the Einstein-Hilbert Lagrangian, causing dynamical terms governing $\varphi$ to emerge due to the non-minimal coupling to $R$.

Equation (45) is the fifth possible scheme, recalling that $\Lambda$ is the vacuum scalar curvature, so if the vacuum curvature is not to contribute in the domain $S$, then $\varepsilon=0$.

Over the entire infinite domain $\Omega$, the variations of $I$ with respect to $g^{\mu v}$ and $\varphi$ of (44) yield,

$$
\begin{gathered}
G_{\mu v}+g_{\mu v} \Lambda=\kappa T_{\mu \nu}-\varphi\left(g_{\mu \nu} g \delta \nabla_{\delta} \nabla_{\gamma}-\nabla_{\mu} \nabla_{v}\right) \frac{1}{\varphi} \\
R-\varphi \frac{\partial R}{\partial \varphi}=2 \kappa\left(\varphi \frac{\partial \mathcal{L}_{m}}{\partial \varphi}-\mathcal{L}_{m}\right)+2 \Lambda \\
\varphi\left(g^{\mu \nu} \nabla_{\mu} \nabla_{v}\right) \frac{1}{\varphi}=\frac{R-4 \Lambda+\kappa T_{\mu}^{\mu}}{3} \\
g_{\mu \nu}\left(\frac{1}{12} R+\frac{\kappa T_{\mu}^{\mu}}{3}-\frac{4 \Lambda}{3}\right)=\kappa T_{\mu \nu}+\varphi \nabla_{\mu} \nabla_{v} \frac{1}{\varphi}
\end{gathered}
$$

where (47) results from the variation of $I$, including the matter action term, with respect to $\varphi$. There are a number of differences between (46) to (49) and the standard form of Brans-Dicke theory with $\omega=0$. Also, the variation is over the infinite domain, not just $S$ or $\Omega-S$, per the eventual goal. In normal General Relativity, the numerator of (48) is exactly zero if the cosmological constant $\Lambda=0$, so the scalar 
field (48) is sourced by any imbalance in its terms from deviations from normal General Relativity. In Brans-Dicke gravity, the scalar field is sourced by the matter stress energy tensor only, and the inverse of the scalar field is the variable G. Note from (46) the scalar field does not divide the stress energy tensor, so it is not $G$ that is varying. The solutions of (46) to (49) for cases of radiation and vacuum have been worked out for homogeneous-isotropic cosmologies, and will be presented in a forthcoming paper.

Note, that in vacuum with no matter fields, and no cosmological constant, the righthand side of (47), derived from (44), is zero. There is a resulting vacuum scalar curvature $R_{\text {vac }}=C \varphi$, an initial motivator for examining this form, as this term could play the role of the Cosmological Constant. A simple way to exclude volume of the field $\varphi$ would be to decompose it as $\varphi=f(r) O$, yielding $R_{v a c}=$ $C f(r) O$. If the partitioning is per (40), there is then a gradient in the vacuum energy, and effectively, there would be an added force. The vacuum (or dark) energy $R_{\text {vac }}$ links the source of dark energy to the alternative explanation for dark matter. The radius of partitioning in (40), and any resulting horizon must be found self-consistently, and will not generally be equal to the usual definition of the Schwarzschild Radius of $2 G M / c^{2}$.

Another motivation for the form of (44), and the related enforced exclusion of a void, is that it was shown by Thorne and also Hawking that the solutions of Brans-Dicke theory for black holes are identical to that of normal General Relativity, and the collapsed mass ceases to be a source for the Brans-Dicke scalar field, becoming constant $[19,20]$, and so the scalar field introduces no change beyond normal General Relativity. Stated another way, the inherent censorship beneath the event horizon in Brans-Dicke theory is not sufficient to result in any differences in behavior acting as alternatives to dark matter. Another motivation is that there are no dynamical terms for the scalar field appearing in the Lagrangian (44), which seems to be a reasonable analogy to the unsourced Newtonian solutions so far discussed. As a result of the latter, the stress energy momentum tensor corresponding to the scalar field $T^{(\varphi)}{ }_{\mu \nu}=0$, and so the scalar field does not act effectively like dark matter-that is, adding to the energy momentum tensor of matter. Yet, one may introduce an intentional non-equality between $R$ and $\kappa T$ along with a metric with sufficient adjustability that leads to a non-constant scalar field satisfying (46) to (49) by self-consistency. The analogy is the use of the many possible forms of Equation (7a-c) for the unsourced Newtonian vacuum potential to fit data.

The form (44) may potentially be applied to any other Lagrangian density within the curly brackets, not just gravity. Such a modification to General Relativity is necessary to attempt to explain the CMB power spectrum features, gravitational lensing, large scale structure formation, the Bullet Cluster, and other observations normally explained by dark matter. It is clear that the rotary charge voids, and therefore rotary fields of this model, are affixed to- and would remain coincident withthe galaxy supermassive black holes during a galaxy cluster collision. The lensing effects would not remain coincident with the gases, as per the same conclusion utilizing dark matter.

Thus, there are at least five schemes for forcibly excluding the event horizon volume, with differing boundary conditions possible for each, and the objective is to find those that do not conflict with what is observed.

The differences between the approaches outlined here, and MOND and TeVeS, is that there are no acceleration dependent form changes, and the metric solutions must be found self-consistently. However, MOND did provide the initial motivation for examining the cases here, as the deep MOND functional form of the field is the same as those of Sections 2 and 3.

\section{Discussion}

It is suggested that the vacuum provides a uniform rotary charge density at all points in space, except beneath the event horizons of black holes at galactic centers, where the vacuum is also suggested to not be able to contribute to a vector cancellation effect. This would also explain why the resultant $1 / r$ dependent force is not observed locally about individual stars, planets, or any object that has not undergone gravitational collapse, as there is no event horizon. As the particles that are responsible for 
the effect are in the vacuum, and it is a lack of their interactions in a void leading to a force, one does not directly detect the particles. This would also explain why the particles do not interact with one another to form elements, compounds, planets, or stars.

How the volume of the vacuum beneath the event horizon becomes a non-contributor at greater range will require elucidation, along with whether the rotary parameters can be made equivalent to a field mediated by particles that are massive or massless. If they are massive, the accumulation of that mass is then not necessarily equivalent to what had been the total mass of galactic dark matter, rather, it is the influence the rotary charge density void that is responsible for the effect.

The question was raised during the submission of this paper whether the vacuum for all possible field species, subject to an excluded volume, would generate a new type of excluded charge. The idea is interesting, and would require more theory as to what the effects might be, such things as: Excluded electromagnetic radiation resulting in a Casimir-like pressure at the event horizon; (2) Excluded electric charges drawing charged objects towards the horizon; etc. The conclusion concerning this question is that there is an upside: If true, there would be multiple effects to look for.

Field Theory and General Relativity are the cornerstones of modern physics. There seem to be some inherent contradictions in both theories. For example, in field theory, a static field functions much like the fields as envisioned by Faraday. Yet, in quantum field theory a static field can be approximated with the tree-level terms of the perturbative expansion to produce an amplitude, with Feynman diagrams showing particle exchange limiting the interaction to the speed of light. When the latter in the non-relativistic limit is equated to the Born approximation amplitude, a classical potential can be computed. Propagation of a field would therefore appear to be required for the static field to function in the higher theory. For a black hole, the mass, charge, and angular momentum are not censored: they are communicated by non-propagating modes in field theory, the accepted explanation. Changes in the static fields are propagated at the speed of light, but once reestablished, are Faraday-like static fields once again, influencing instantaneously at a distance. Physical constants would be static fields, and like any static field, described by non-propagating modes, again Faraday-like, influencing instantaneously at a distance. When matter or charges gravitate into the event horizon of a black hole, the initial non-propagating modes, understood to have been set up long ago, quickly readjust, to produce the new non-propagating modes. Yet, despite the censorship of the event horizon, somehow, in the particle picture, particles (fields) must propagate from the event horizon to reset the non-propagating modes - thus emission of particles from the horizon would seem to be needed. The descriptions conflict, despite the predictive power. As to General Relativity, it is perfectly acceptable at this time that energy and momentum are not conserved when there are dynamical changes in spacetime, although how the non-conservation evolves is well understood, with the conservation possible only in a locally flat frame. With a cosmological constant, the total energy of the universe increases (explained by negative pressure). The variation of physical constants throughout the universe may also constitute acceptable violations of conservation laws. The field $\varphi$ in (44) and (45) may be representative of a varying constant.

In the model presented here, part of the influence of a black hole is explained by the properties of what remains outside of the event horizon, not by what the black hole has digested, and so avoids altogether conflicts with how effects are communicated outward.

\section{Conclusions}

It is merely noted that flat galaxy rotation curves are admitted by the physics of the era of Poisson and Laplace, without added dark matter distributions, and without direct relation to the gravitational constant. Poisson and Laplace did not know of galaxy rotation curves, dark matter or energy, General Relativity, vacuum polarization, or black holes, but might have surmised that a spatially confined absence of a form of charge might alter Newtonian gravity using homogeneous solutions.

The classical vacuum is here made to generate communicable forces. Insight is offered as to why such a field term - that is the rotational charge $q$-would become important on the galactic scale only, and is due to the presence of a supermassive blackhole that prevents the event horizon volume, of 
what would otherwise be an uninterrupted distribution, from interacting. Boundary conditions for the five schemes outlined for the General Relativistic case will be developed in further work.

Appendices A and B follow below, discussing gravitational vector potentials, and showing that gravitomagnetism is not admitted by the field under study.

Funding: Research funding was provided by Optical Physics Company, Simi Valley, CA.

Conflicts of Interest: The author declares no conflict of interest.

\section{Appendix A. Rotary Vector Potential in Cylindrical Coordinates}

The vector potential to be discussed is not related to the gravitomagnetic field, and is further suggestive of a $2 \mathrm{D}$ or cylindrical character. Consider the addition of the curl of a vector potential $f$ to the field to satisfy the equivalent of the vacuum condition (6),

$$
\begin{aligned}
& \nabla \cdot \bar{g}=-4 \pi G \rho \\
& \psi=\psi_{\text {mat }}+\psi_{\text {curl }} \\
& \bar{g}=-\nabla \psi \rightarrow-\nabla \psi_{\text {mat }}-\nabla \psi_{\text {curl }}=-\nabla \psi_{\text {mat }}+\nabla \times \bar{f} \\
& \nabla^{2} \psi_{\text {curl }}=-\nabla \cdot(\nabla \times \bar{f})=0 \\
& \nabla^{2} \psi=\nabla^{2} \psi_{\text {mat }}=4 \pi G \rho \\
& \nabla \psi_{\text {curl }}=-\nabla \times \bar{f}
\end{aligned}
$$

While the curl of the gradient of a scalar field is zero, it is possible to equate the curl of a vector field to the gradient of a scalar field. The condition (A1f) for the desired logarithmic potential is satisfied non-uniquely, and also with gauge symmetry, with solutions found most easily in the cylindrically symmetric case, examples being,

$$
\begin{aligned}
& \nabla(q \ln (r / a))=\frac{q}{r} \hat{r}=-\nabla \times \bar{f} \\
& -\nabla \times \bar{f}_{c y l}=\left\{\begin{array}{c}
-\frac{\hat{r}}{r} \frac{\partial f_{z}}{\partial \varphi} \\
\hat{r} \frac{\partial f_{\varphi}}{\partial z}
\end{array}\right. \\
& \bar{f}_{c y l}=\left\{\begin{array}{c}
-q \varphi \hat{z}+\nabla \xi \\
q z / r \hat{\varphi}+\nabla \xi
\end{array}\right.
\end{aligned}
$$

where $\xi$ is any scalar function. There are no obvious solutions for the spherically symmetric case, as Equation (A1a) does not hold, recalling (17). The vector potentials (A2c) are shown in Figure A1 for zero $\xi$.

Therefore, the condition (5) is upheld for the vector potentials (A2c). The curls of the vector potentials are equal to the gradients of scalar potentials, and the scalar potentials are equivalent to vacuum potentials, as their Laplacian's are zero.

The field $g$ then has two components obeying (5) and in cylindrical coordinates,

$$
\begin{gathered}
\bar{g}=\bar{g}_{\rho}+\bar{g}_{q} \\
\nabla \cdot \bar{g}_{\rho}=-4 \pi G \rho \\
\nabla \cdot \bar{g}_{q}=0 \\
\bar{g}_{q}=\nabla \times \bar{f}
\end{gathered}
$$



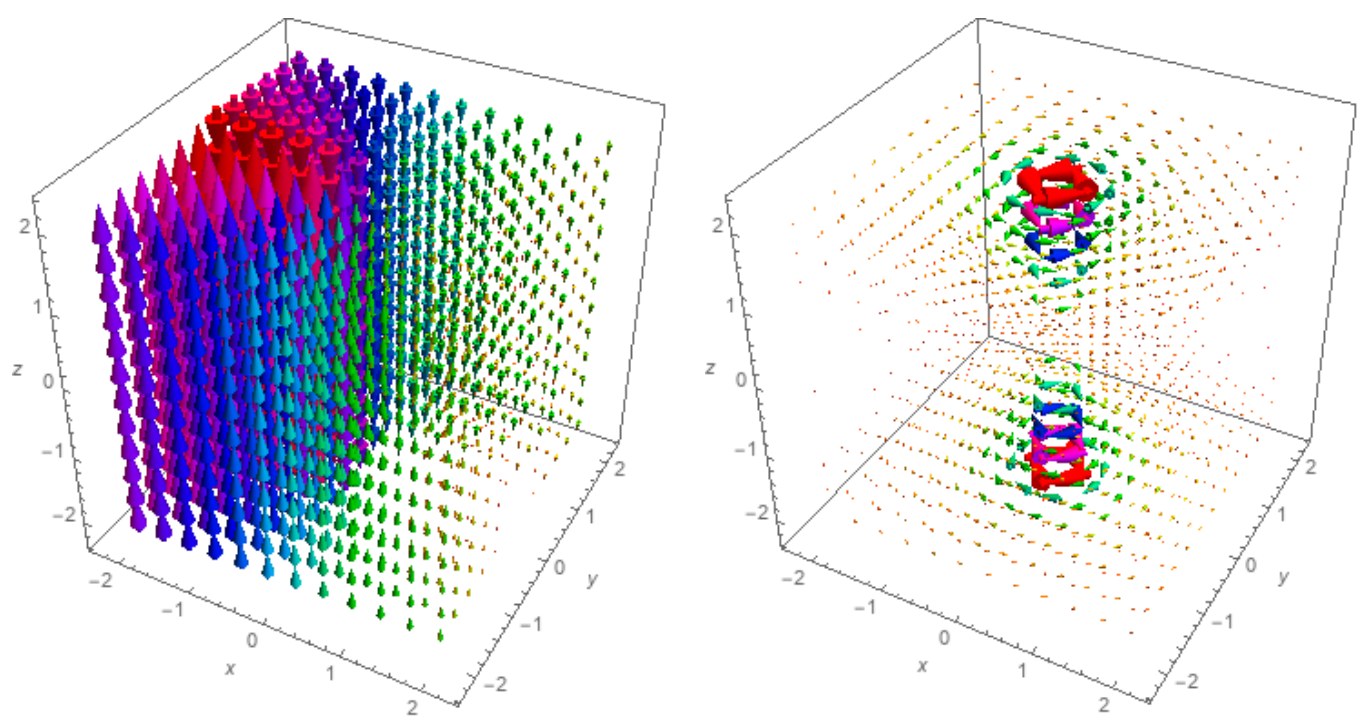

Figure A1. Vector potentials $f$ of Equation (A2c) plotted in Cartesian coordinates. (Left) Top solution.

(Right) Bottom solution. The rotary charge $q=1$, axis units are arbitrary. The scalar field $\xi$ is zero.

\section{Appendix B. Comment on Gravitomagnetic Field}

The condition (5) limits the solutions $g$ to be time independent, if one were to attempt an analogy to Maxwell's equations in vacuum, as:

$$
\begin{aligned}
& \nabla \cdot \bar{g}_{\rho}=0 \\
& \nabla \cdot \bar{g}_{q}= \begin{cases}0 & c y l . \\
-q / r^{2} & \text { sph. }\end{cases} \\
& \nabla \times \bar{g}_{\rho}=-\frac{1}{k} \frac{\partial \bar{g}_{q}}{\partial t}=0 \\
& \nabla \times \bar{g}_{q}=\frac{1}{k} \frac{\partial \bar{g}_{\rho}}{\partial t}=0
\end{aligned}
$$

Since (A4(c,d)) must be zero, the fields are static, and this restates that the vector potential $f$ is unrelated to the gravitomagnetic field.

\section{References}

1. Akerib, D.S.; Alsum, S.; Araújo, H.M.; Bai, X.; Bailey, A.J.; Balajthy, J.; Beltrame, P.; Bernard, E.P.; Bernstein, A.; Biesiadzinski, T.P.; et al. Results from a Search for Dark Matter in the Complete LUX Exposure. Phys. Rev. Lett. 2017, 118, 021303. [CrossRef] [PubMed]

2. Aprile, E.; Aalbers, J.; Agostini, F.; Alfonsi, M.; Amaro, F.D.; Anthony, M.; Arneodo, F.; Barrow, P.; Baudis, L.; Bauermeister, B.; et al. First Dark Matter Search Results from the XENON1T Experiment. Phys. Rev. Lett. 2017, 119, 181301. [CrossRef] [PubMed]

3. Cui, X.; Abdukerim, A.; Chen, W.; Chen, X.; Chen, Y.; Dong, B.; Fang, D.; Fu, C.; Giboni, K.; Giuliani, F.; et al. Dark Matter Results From 54-Ton-Day Exposure of Panda X-II Experiment. arXiv 2017, arXiv:1708.06917.

4. Ackermann, M.; Ajello, M.; Albert, A.; Atwood, W.B.; Baldini, L.; Ballet, J.; Barbiellini, G.; Bastieri, D.; Bellazzini, R.; Bissaldi, E.; et al. The Fermi Galactic Center GeV Excess and Implications for Dark Matter. Astrophys. J. 2017, 840, 43. [CrossRef]

5. Dicus, D.A.; Kolb, E.W.; Teplitz, V.L.; Wagoner, R.V. Astrophysical bounds on the masses of axions and Higgs particles. Phys. Rev. D 1978, 18, 1829-1834. [CrossRef]

6. Dicus, D.A.; Kolb, E.W.; Teplitz, V.L.; Wagoner, R.V. Astrophysical bounds on very-low-mass axions. Phys. Rev. D 1980, 22, 839-845. [CrossRef] 
7. Asztalos, S.J.; Carosi, G.; Hagmann, C.; Kinion, D.; Van Bibber, K.; Hotz, M.; Rosenberg, L.J.; Rybka, G.; Hoskins, J.; Hwang, J.; et al. SQUID-Based Microwave Cavity Search for Dark-Matter Axions. Phys. Rev. Lett. 2010, 104, 041301. [CrossRef] [PubMed]

8. Du, N.; Force, N.; Khatiwada, R.; Lentz, E.; Ottens, R.; Rosenberg, L.J.; Rybka, G.; Carosi, G.; Woollett, N.; Bowring, D.; et al. Search for Invisible Axion Dark Matter with the Axion Dark Matter Experiment. Phys. Rev. Lett. 2018, 120, 151301. [CrossRef] [PubMed]

9. Moffat, J.W. Scalar-Tensor-Vector Gravity Theory. J. Cosmol. Astropart. Phys. 2006, 3, 4. [CrossRef]

10. Buchdahl, H.A. Non-linear Lagrangians and cosmological theory. Mon. Not. R. Astron. Soc. 1970, 150, 1-8. [CrossRef]

11. Bekenstein, J.D. Relativistic gravitation theory for the modified Newtonian dynamics paradigm. Phys. Rev. D 2004, 70, 083509. [CrossRef]

12. Milgrom, M. A modification of the Newtonian dynamics as a possible alternative to the hidden mass hypothesis. Astrophys. J. 1983, 270, 365-370. [CrossRef]

13. Famaey, B.; McGaugh, S. Modified Newtonian Dynamics (MOND): Observational Phenomenology and Relativistic Extensions. Living Rev. Relativ. 2012, 15, 10. [CrossRef] [PubMed]

14. Kuhn, J.R.; Kruglyak, L. Non-Newtonian Forces and the Invisible Mass Problem. Astrophys. J. 1987, 131, 1-12. [CrossRef]

15. Zhang, F. Modeling the Rising Tails of Galaxy Rotation Curves. Galaxies 2019, 7, 27. [CrossRef]

16. Pisani, A.; Massara, E.; Spergel, D.N.; Alonso, D.; Baker, T.; Cai, Y.-C.; Cautun, M.; Davies, C.; Demchenko, V.; Doré, O.; et al. Cosmic voids: A novel probe to shed light on our Universe. arXiv Preprint 2019, arXiv:1903.05161.

17. Cautun, M.; Paillas, E.; Cai, Y.-C.; Bose, S.; Armijo, J.; Li, B.; Padilla, N. The Santiago-Harvard-EdinburghDurham void comparison-I. SHEDding light on chameleon gravity tests. Mon. Not. R. Astron. Soc. 2018, 476, 3195-3217. [CrossRef]

18. Gurtug, O.; Mangut, M.; Halilsoy, M. Gravitational Lensing in Rotating and Twisting Universes. arXiv Preprint 2020, arXiv:2002.00042.

19. Thorne, K.S.; Dykla, J.J. Black Holes in the Dicke-Brans-Jordan Theory of Gravity. Astrophys. J. 1971, 166, L35-L38. [CrossRef]

20. Hawking, S.W. Black Holes in the Brans-Dicke Theory of Gravity. Commun. Math. Phys. 1972, 25, 161-162. [CrossRef] 